\title{
Testing the No-Hair Theorem with GW150914
}

\author{
Maximiliano Isi® ${ }^{1,{ }^{*}}$ Matthew Giesler, ${ }^{2}$ Will M. Farr, ${ }^{3,4}$ Mark A. Scheel, ${ }^{2}$ and Saul A. Teukolsky ${ }^{2,5}$ \\ ${ }^{1}$ LIGO Laboratory, Massachusetts Institute of Technology, Cambridge, Massachusetts 02139, USA \\ ${ }^{2}$ TAPIR, Walter Burke Institute for Theoretical Physics, California Institute of Technology, Pasadena, California 91125, USA \\ ${ }^{3}$ Center for Computational Astrophysics, Flatiron Institute, 162 5th Avenue, New York, New York 10010, USA \\ ${ }^{4}$ Department of Physics and Astronomy, Stony Brook University, Stony Brook, New York 11794, USA \\ ${ }^{5}$ Cornell Center for Astrophysics and Planetary Science, Cornell University, Ithaca, New York 14853, USA
}

(Received 5 May 2019; published 12 September 2019)

\begin{abstract}
We analyze gravitational-wave data from the first LIGO detection of a binary black-hole merger (GW150914) in search of the ringdown of the remnant black hole. Using observations beginning at the peak of the signal, we find evidence of the fundamental quasinormal mode and at least one overtone, both associated with the dominant angular mode $(\ell=m=2)$, with $3.6 \sigma$ confidence. A ringdown model including overtones allows us to measure the final mass and spin magnitude of the remnant exclusively from postinspiral data, obtaining an estimate in agreement with the values inferred from the full signal. The mass and spin values we measure from the ringdown agree with those obtained using solely the fundamental mode at a later time, but have smaller uncertainties. Agreement between the postinspiral measurements of mass and spin and those using the full waveform supports the hypothesis that the GW150914 merger produced a Kerr black hole, as predicted by general relativity, and provides a test of the no-hair theorem at the $\sim 10 \%$ level. An independent measurement of the frequency of the first overtone yields agreement with the no-hair hypothesis at the $\sim 20 \%$ level. As the detector sensitivity improves and the detected population of black-hole mergers grows, we can expect that using overtones will provide even stronger tests.
\end{abstract}

DOI: 10.1103/PhysRevLett.123.111102

Introduction.-The coalescence of two astrophysical black holes consists of a long inspiral followed by a violent plunge, during which the full richness of spacetime dynamics comes into play. The two objects merge, forming a single distorted black hole that rings down as it settles to a final stationary state. Gravitational waves are emitted throughout the entire process, at each moment carrying information about the evolving source. In general relativity, radiation from the ringdown stage takes the form of superposed damped sinusoids, corresponding to the quasinormal-mode oscillations of the final Kerr black hole [1-4]. The frequencies and decay rates of these damped sinusoids are uniquely determined by the final hole's mass $M_{f}$ and dimensionless spin magnitude $\chi_{f}$. This is a consequence of the no-hair theorem - the statement that mass and spin are the only two properties of astrophysical black holes in general relativity. (In general, black holes may also possess electric charge, but this is expected to be negligible for astrophysical objects.) The ringdown spectrum is thus a fingerprint that identifies a Kerr black hole: measuring the quasinormal modes from gravitational-wave observations would provide us with a unique laboratory to test general relativity and probe the true nature of remnants from compact-binary mergers, including testing the no-hair theorem [5-13]. This program has been called black-hole spectroscopy, in analogy to the spectroscopic study of atomic elements [6].
Although LIGO [14] and Virgo [15] have already confidently detected gravitational waves from multiple binary-black-hole coalescences [16-22], black-hole spectroscopy has remained elusive [23-29]. This is because past analyses looked for the ringdown in data at late times after the signal peak, where the quasinormal modes are too weak to confidently characterize with current instruments. The choice to focus on the late, weak-signal regime stemmed from concerns about nonlinearities surrounding the black-hole merger, which were traditionally expected to contaminate the ringdown measurement at earlier times [8,25,26,28-31].

Concerns about nonlinearities are, however, unfounded: the linear description can be extended to the full waveform following the peak of the gravitational wave strain [32]. Rather than nonlinearities, times around the peak are dominated by ringdown overtones - the quasinormal modes with the fastest decay rates, but also the highest amplitudes near the waveform peak [32,33]. Indications of this can be found in the waveform modeling literature, with overtones an integral part of earlier equivalent one-body models [34-36] (although later abandoned, cf., Ref. [37]). Yet, with a few exceptions [12,27], previous ringdown analyses have neglected overtones, under the assumption that their contribution to the signal should always be marginal $[8,23-26,28,29,38]$. As a consequence, these 
studies ignored important signal content and were unable to extract multiple ringdown modes.

The inclusion of overtones enables us to perform a multimodal spectroscopic analysis of a black-hole ringdown, which we apply to LIGO data from the GW150914 event [16] (Fig. 1). We rely on overtones of the $\ell=m=2$ angular mode to measure the remnant mass and spin from data starting at the peak of the signal, assuming first that quasinormal modes are as predicted for a Kerr black hole within general relativity. We find the least-damped ("fundamental") mode and at least one overtone with $3.6 \sigma$ confidence (Fig. 2). At least one overtone, in addition to the fundamental, is needed to describe the waveform near the peak amplitude. This agrees with our expectations from Ref. [32] given the signal-to-noise ratio of GW150914.

Assuming the remnant is a Kerr black hole, frequencies and damping rates of the fundamental mode and one overtone imply a detector-frame mass of $(68 \pm 7) M_{\odot}$

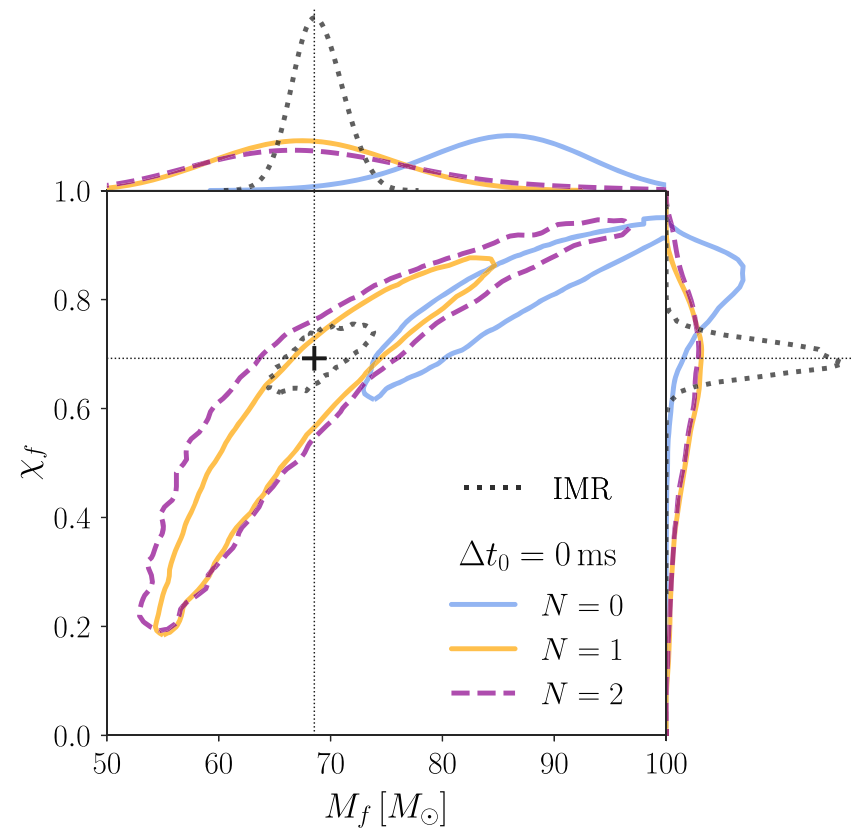

FIG. 1. Remnant parameters inferred with different number of overtones, using data starting at peak strain amplitude. Contours represent $90 \%$-credible regions on the remnant mass $\left(M_{f}\right)$ and dimensionless spin magnitude $\left(\chi_{f}\right)$, obtained from the Bayesian analysis of GW150914. The inference model is that of Eq. (1), with different number of overtones $N$ : 0 (solid blue), 1 (solid yellow), 2 (dashed purple). In all cases, the analysis uses data starting at peak strain $\left(\Delta t_{0}=t_{0}-t_{\text {peak }}=0\right)$. Amplitudes and phases are marginalized over. The black contour is the $90 \%$ credible region obtained from the full IMR waveform, as described in the text. The intersection of the dotted lines marks the peak of this distribution $\left(M_{f}=68.5 M_{\odot}, \chi_{f}=0.69\right)$. The top and right-hand panels show $1 \mathrm{D}$ posteriors for $M_{f}$ and $\chi_{f}$, respectively. The linear quasinormal mode models with $N>0$ provide measurements of the mass and spin consistent with the full IMR waveform, in agreement with general relativity. and a dimensionless spin magnitude of $0.63 \pm 0.16$, with $68 \%$ credibility. This is the best constraint on the remnant mass and spin obtained in this work. This measurement agrees with the one obtained from the fundamental mode alone beginning $3 \mathrm{~ms}$ after the waveform peak amplitude (Figs. 1 and 3) [39]. It also agrees with the mass and spin inferred from the full waveform using fits to numerical relativity. The fractional difference between the bestmeasured combination of mass and spin at the peak with one overtone and the same combination solely with the fundamental $3 \mathrm{~ms}$ after the peak is $(0 \pm 10) \%$ [40]. This is evidence at the $\sim 10 \%$ level that GW150914 did result in a Kerr black hole as predicted by general relativity, and that the postmerger signal is in agreement with the no-hair theorem. Similarly, the fractional difference between the best-measured combination of mass and spin at the peak with one overtone and the same combination using the full waveform is $(7 \pm 7) \%$.

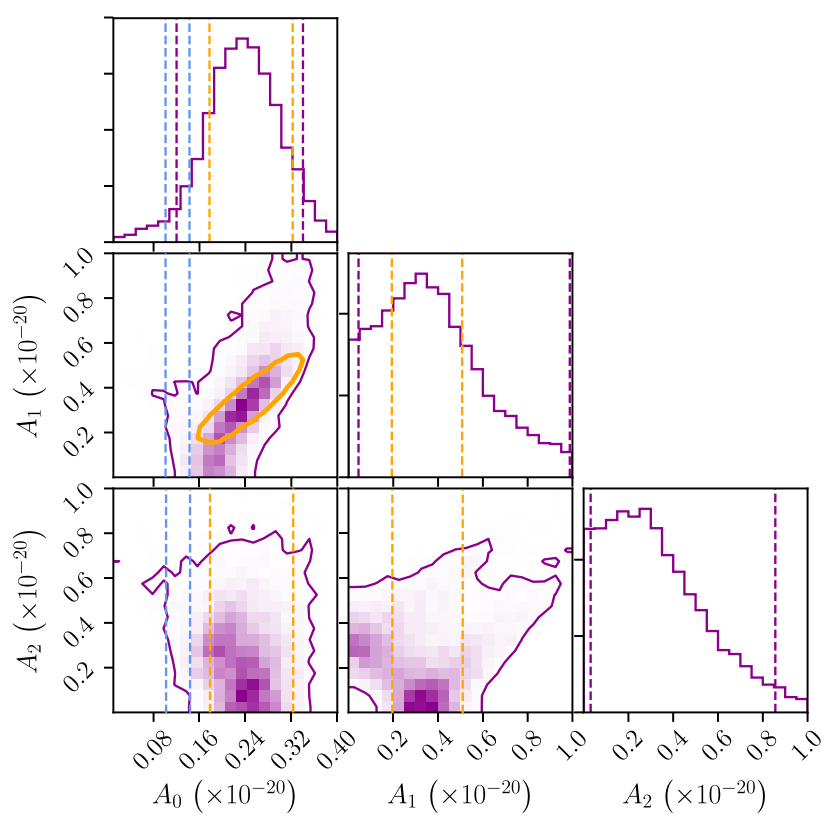

FIG. 2. Measured quasinormal-mode amplitudes for a model with the fundamental mode and two overtones $(N=2)$. The purple color map represents the joint posterior distribution for the three amplitudes in the $N=2$ model: $A_{0}, A_{1}, A_{2}$, as defined in Eq. (1). The solid curves enclose $90 \%$ of the probability mass. A yellow curve in the $A_{0}-A_{1}$ plane, as well as corresponding yellow dashed lines, represents the $90 \%$-credible measurement of the amplitudes assuming $N=1$. Similarly, blue dashed lines give the $90 \%$-credible measurement of $A_{0}$ assuming $N=0$. All amplitudes are defined at $t=t_{\text {peak }}$, where all fits here are carried out $\left(\Delta t_{0}=0\right)$. Values have been rescaled by a constant to correspond to the strain measured by the LIGO Hanford detector. Assuming $N=1$, the mean of the $A_{1}$ marginalized posterior lies 3.6 standard deviations away from zero; i.e., $A_{1}=0$ is disfavored at 3.6 $\sigma$. Assuming $N=2, A_{1}=A_{2}=0$ is disfavored with $90 \%$ credibility. 


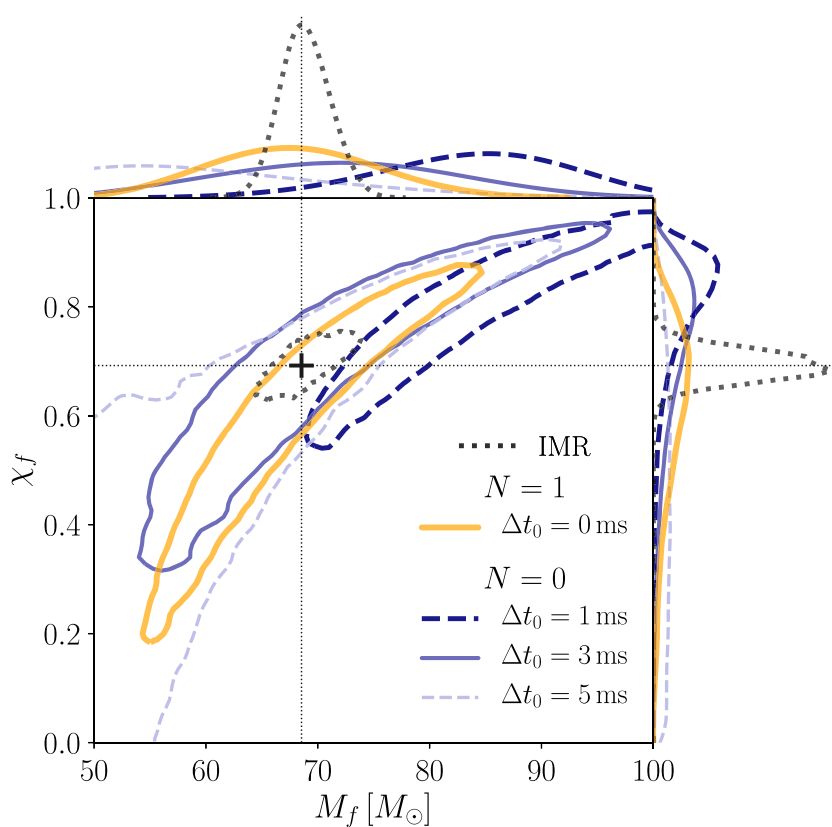

FIG. 3. Remnant parameters inferred only from the fundamental mode, using data starting at different times after the peak. Contours represent $90 \%$-credible regions on the remnant mass $\left(M_{f}\right)$ and dimensionless spin magnitude $\left(\chi_{f}\right)$, obtained from the Bayesian analysis of GW150914. For the blue contours, the inference model included no overtones $(N=0)$ and used data starting at different times after the peak: $\Delta t_{0}=t_{0}-t_{\text {peak }} \in[1,3,5] \mathrm{ms}$. For the yellow contour, the analysis was conducted with one overtone $(N=1)$ starting at the peak $\left(\Delta t_{0}=0\right)$, as in Fig. 1. Amplitudes and phases are marginalized over. The black contour is the $90 \%$ credible region obtained from the full IMR waveform, as described in the text. The intersection of the dotted lines marks the peak of this distribution $\left(M_{f}=68.5 M_{\odot}, \chi_{f}=0.69\right)$. The top and righthand panels show $1 \mathrm{D}$ posteriors for $M_{f}$ and $\chi_{f}$, respectively. Around $\Delta t_{0}=3 \mathrm{~ms}$, the overtones have become unmeasurable and only the fundamental mode remains; consequently, at that time $N=0$ returns a measurement of the final mass and spin consistent with both the full IMR waveform and the $N>0$ models at the peak, in agreement with general relativity.

Traditional proposals for black-hole spectroscopy require frequency measurements for two or more quasinormal modes [6]. In that spirit, we also consider a singleovertone model that allows the overtone frequency and damping time to deviate from the Kerr prediction for any given mass and spin. This enables us to evaluate the agreement of the observed ringdown spectrum with the prediction for a perturbed Kerr black hole, regardless of the specific properties of the remnant. From analysis of data starting at peak strain, we find the spectrum to be in agreement with the no-hair hypothesis to within $20 \%$, with $68 \%$ credibility (Fig. 4). This is a test of the no-hair theorem based purely on the postinspiral regime.

Method.-Each quasinormal mode has a frequency $\omega_{\ell m n}$ and a damping time $\tau_{\ell m n}$, where $n$ is the "overtone" index and $(\ell, m)$ are indices of spin-weighted angular harmonics

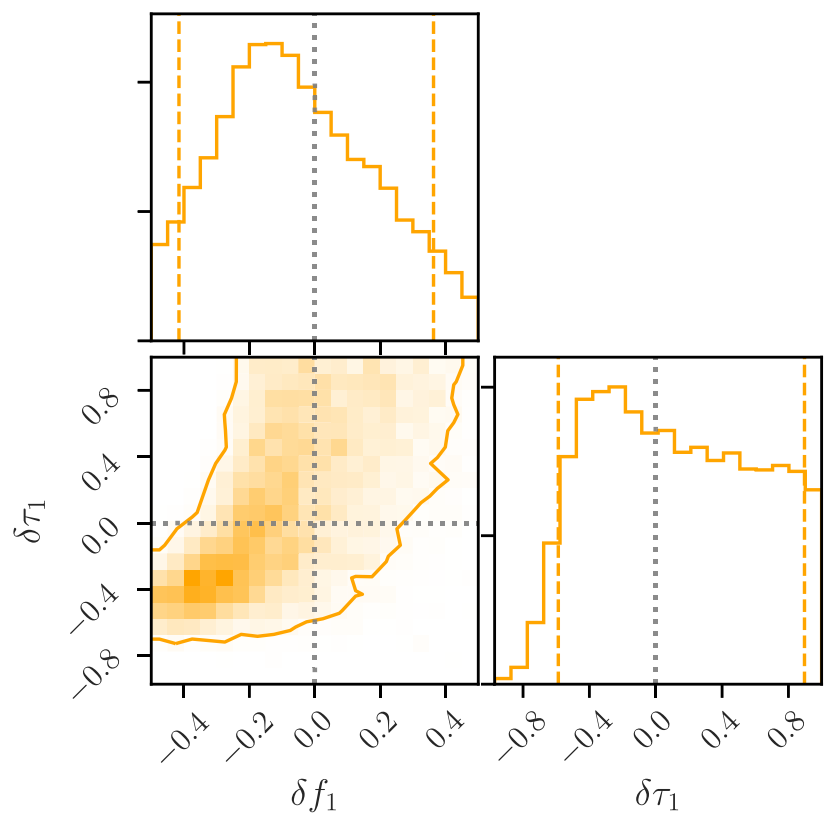

FIG. 4. Measurement of the frequency and damping time of the first overtone, using data starting at the peak. The color map represents the posterior distribution of the fractional deviations $\delta f_{1}$ and $\delta \tau_{1}$ away from the no-hair value $\delta f_{1}=\delta \tau_{1}=0$ (gray dotted lines). The solid contour and dashed vertical lines enclose $90 \%$ of the posterior probability. All other parameters, including $M_{f}$ and $\chi_{f}$, have been marginalized away. Fixing $\delta f_{1}=\delta \tau_{1}=0$ recovers the $N=1$ analysis in Figs. 1 and 3 .

that describe the angular dependence of the mode. We focus on the fundamental and overtones of the dominant $\ell=m=2$ spin-weighted spherical harmonic of the strain [41]. This is the only angular harmonic expected to be relevant for GW150914 [45,46]. (Dedicated studies have found no evidence of higher angular harmonics in the late ringdown of GW150914 [29].) For ease of notation, we generally drop the $\ell$ and $m$ indices, retaining only the overtone index $n$. The $\ell=m=2$ mode of the parametrized ringdown strain $\left(h=h_{+}-i h_{\times}\right)$can be written as a sum of damped sinusoids [1-4],

$$
h_{22}^{N}(t)=\sum_{n=0}^{N} A_{n} \exp \left[-i\left(\omega_{n} t+\phi_{n}\right)-t / \tau_{n}\right],
$$

for times $t$ greater than some start time $t_{0}$, where $\Delta t=$ $t-t_{0}$. The overtone index $n$ orders the different modes by decreasing damping time $\tau_{n}$, so that $n=0$ denotes the longest-lived mode. $N$ is the index of the highest overtone included in the model, which in this work will be $N \leq 2$. Importantly, higher $n$ does not imply a higher frequency $\omega_{n}$; rather, the opposite is generally true. All frequencies and damping times are implicit functions of the remnant mass and spin magnitude $\left(M_{f}, \chi_{f}\right)$, and can be computed from perturbation theory [47-49]. The amplitudes $A_{n}$ and phases $\phi_{n}$ encode the degree to which each overtone is 
excited as the remnant is formed and cannot be computed within perturbation theory, so we treat them as free parameters in our fit.

We use the model in Eq. (1) to carry out a Bayesian analysis of LIGO Hanford and LIGO Livingston data for GW150914 [16,22,50,51]. For any given start time $t_{0}$, we produce a posterior probability density over the space of remnant mass and spin magnitude, as well as the amplitudes and phases of the included overtones. We parametrize start times via $\Delta t_{0}=t_{0}-t_{\text {peak }}$, where $t_{\text {peak }}=$ 1126259462.423 (global positioning system time) refers to the inferred signal peak at the LIGO Hanford detector $[23,52]$. We define the likelihood in the time domain in order to explicitly exclude all data before $t_{0}$. We place uniform priors on $\left(M_{f}, \chi_{f}, A_{n}, \phi_{n}\right)$, with a restriction to corotating modes $\left(\omega_{n}>0\right)$. All overtones we consider share the same $\ell=m=2$ angular dependence, allowing us to simplify the handling of antenna patterns and other subtleties. Details specific to our implementation are provided in the Supplemental Material [53].

We compare our ringdown-only measurements of the remnant mass and spin magnitude to those obtained from the analysis of the full inspiral-merger-ringdown (IMR) signal. To do so, we rely on fitting formulas based on numerical relativity to translate measured values of the binary mass ratio $q$ and component spins $\left(\vec{\chi}_{1}, \vec{\chi}_{2}\right)$ into expected remnant parameters $[54,55]$. We use posterior samples on the binary parameters made available by the LIGO and Virgo Collaborations [22,56], marginalizing over unavailable component-spin angles.

We consider explicit deviations from the Kerr spectrum by allowing the frequency and damping time of the first overtone to differ from the no-hair values. Under this modified $N=1$ model, the overtone angular frequency becomes $\omega_{1}=2 \pi f_{1}^{(\mathrm{GR})}\left(1+\delta f_{1}\right)$, with $\delta f_{1}$ a fractional deviation away from the Kerr frequency $f_{1}^{(\mathrm{GR})}$ for any given $M_{f}$ and $\chi_{f}$. Similarly, the damping time is allowed to vary by letting $\tau_{1}=\tau_{1}^{(\mathrm{GR})}\left(1+\delta \tau_{1}\right)$. Fixing $\delta f_{1}=\delta \tau_{1}=0$ recovers the regular $N=1$ analysis. We may then compute the relative likelihood of the no-hair hypothesis by means of the Savage-Dickey density ratio [57].

Results.-Figure 1 shows the 90\%-credible regions for the remnant mass (abscissa) and spin magnitude (ordinate) obtained by analyzing data starting at $t_{\text {peak }}$ with different numbers of overtones $(N=0,1,2)$ in the ringdown template of Eq. (1). The quasinormal-mode amplitudes and phases have been marginalized over. For comparison, we also show the $90 \%$-credible region inferred from the full IMR signal, as explained above. If the remnant is sufficiently well described as a perturbed Kerr black hole, and if general relativity is correct, we expect the ringdown and IMR measurements to agree. As expected, this is not the case if we assume the ringdown is composed solely of the longest-lived mode $(N=0)$, in which case we obtain a biased estimate of the remnant properties. In contrast, the ringdown and IMR measurements begin to agree with the addition of one overtone $(N=1)$. This is expected from previous work suggesting that, given the network signal-tonoise ratio of GW150914 ( 14 in the postpeak region, for frequencies $>154.7 \mathrm{~Hz}$ ), we should be able to resolve only one mode besides the fundamental [32].

Indeed, a ringdown model with two overtones $(N=2)$ does not lead to further improvement in the mass and spin measurement. On the contrary, the $90 \%$-credible region obtained with $N=2$ is slightly broader than the one with $N=1$, as might be expected from the two additional free parameters $\left(A_{2}, \phi_{2}\right)$. This is because the analysis is unable to unequivocally identify the second overtone in the data, as shown by the amplitude posteriors in Fig. 2. The $N=2$ posterior supports a range of values for $A_{1}$ and $A_{2}$, but excludes $A_{1}=A_{2}=0$ with $90 \%$ credibility (center panel in bottom row of Fig. 2). The joint posterior distribution on $A_{1}$ and $A_{2}$ tends to favor the first overtone at the expense of the second: the maximum a posteriori waveform scarcely includes any contribution from $n=2$, and favors a value of $A_{1}$ in agreement with the $N=1$ posterior (yellow traces in Fig. 2).

We next compare measurements carried out with overtones at the peak with measurements without overtones after the peak. Figure 3 shows 90\%-credible regions for the remnant mass and spin magnitude obtained with the fundamental mode $(N=0)$ at different times after $t_{\text {peak }}\left(\Delta t_{0} \in[1,3,5] \mathrm{ms}\right)$. As the overtones die out, the fundamental mode becomes a better model for the signal. We find that the $N=0$ contour coincides with the IMR measurement $\sim 3 \mathrm{~ms}$ after the peak, in agreement with Ref. [23]. However, the uncertainty in this measurement is larger than for the $N=1$ contour at the peak (also shown for reference). This can be attributed to the exponential decrease in signal-to-noise ratio for times after the peak.

Finally, we allow the first-overtone frequency and damping time to float around the no-hair values in an $N=1$ model. As in Fig. 1, we analyze data starting at the inferred peak of the strain. Figure 4 shows the resulting marginalized posterior over the fractional frequency and damping time deviations ( $\delta f_{1}$ and $\delta \tau_{1}$, respectively). With $68 \%$ credibility, we measure $\delta f_{1}=-0.05 \pm 0.2$. To that level of credibility, this establishes agreement with the nohair hypothesis $\left(\delta f_{1}=0\right)$ at the $20 \%$ level. The damping time is largely unconstrained in the $-0.06 \lesssim \delta \tau_{1} \lesssim 1$ range. This has little impact on the frequency measurement, which is unaffected by setting $\delta \tau_{1}=0$. We find that the ratio of marginal likelihoods (the Bayes factor) between the no-hair model $\left(\delta f_{1}=\delta \tau_{1}=0\right)$ and our floating frequency and damping time model is 1.75 .

Discussion and prospects.-A linearly perturbed Kerr black hole radiates gravitational waves in the form of damped sinusoids, with specific frequencies and decay 
rates determined exclusively by the hole's mass and spin. For any given angular harmonic, the quasinormal modes can be ordered by decreasing damping time through an overtone index $n$, with $n=0$ denoting the longest-lived mode (also known as the fundamental). Although modes of all $n$ contribute to the linear description, the fundamental has long been the only one taken into account in observational studies of the ringdown, with overtones virtually ignored [23-26,28,29]. Yet, these short-lived modes can dominate the gravitational wave signal for times around the peak and are an essential part of the ringdown [32,33]. We demonstrate this with a multimode analysis of the GW150914 ringdown.

Making use of overtones, we extract information about the GW150914 remnant using only postinspiral data, starting at the peak of the signal (Fig. 1). We find evidence of the fundamental mode plus at least one overtone (Fig. 2), and obtain a $90 \%$-credible measurement of the remnant mass and spin magnitude in agreement with that inferred from the full waveform. This measurement is also consistent with the one obtained using solely the fundamental mode at a later time, but has reduced uncertainties (Fig. 3).

The agreement between all measurements is evidence that, beginning as early as the signal peak, a far-away observer cannot distinguish the source from a linearly perturbed Kerr background with a fixed mass and spin; i.e., we do not observe nonlinearities in this regime. The agreement between the IMR and postmerger estimates implies that the data agree with the full prediction of general relativity. This is similar to the consistency test between inspiral and merger ringdown [58,59], but relies on a manifestly linear description of the postinspiral signal. More specifically, it validates the prediction for the final state of a collision between two black holes.

With the identification of multiple ringdown modes, this is also a step toward the goal of black-hole spectroscopy. The agreement between postinspiral measurements with two different sets of modes (Fig. 3) supports the hypothesis that GW150914 produced a Kerr black hole as described by general relativity. Moreover, we constrain deviations away from the no-hair spectrum by allowing the overtone frequency and damping time to vary freely (Fig. 4). This is equivalent to independently measuring the frequencies of the fundamental and first overtone, and establishing their consistency with the Kerr hypothesis.

Future studies of black-hole ringdowns relying on overtones could potentially allow us to identify black-hole mimickers and probe the applicability of the no-hair theorem with high precision, even with existing detectors. Such advances will be facilitated by improvements in our understanding of how the overtones are sourced, so that we can predict the amplitudes and phases from the binary properties. This would reduce the dimensionality of the problem and lead to more specific predictions from general relativity.
This research has made use of data, software, and/or Web tools obtained from the Gravitational Wave Open Science Center [50,56], a service of the LIGO Laboratory, the LIGO Scientific Collaboration, and the Virgo Collaboration.

We thank Aaron Zimmerman for valuable feedback. We thank Gregorio Carullo, Walter del Pozzo, and John Veitch for discussions of their paper on and methods for timedomain analysis [29]. We thank Alessandra Buonanno for clarifications on past use of quasinormal and pseudoquasinormal ringdown modes in waveform modeling. M. I. is supported by NASA through the NASA Hubble Fellowship Grant No. HST-HF2-51410.001-A awarded by the Space Telescope Science Institute, which is operated by the Association of Universities for Research in Astronomy, Inc., for NASA, under Contract No. NAS5-26555. M. I. is a member of the LIGO Laboratory. LIGO was constructed by the California Institute of Technology and Massachusetts Institute of Technology with funding from the National Science Foundation and operates under Cooperative Agreement No. PHY-0757058. M. G. and M. A. S. are supported by the Sherman Fairchild Foundation and NSF Grants No. PHY-1708212 and No. PHY-1708213 at Caltech. S. A. T. is supported in part by the Sherman Fairchild Foundation and by NSF Grants No. PHY1606654 and No. ACI-1713678 at Cornell. The Flatiron Institute is supported by the Simons Foundation. This paper carries LIGO Document No. LIGO-P1900135.

*maxisi@mit.edu

[1] C. V. Vishveshwara, Phys. Rev. D 1, 2870 (1970).

[2] W. H. Press, Astrophys. J. 170, L105 (1971).

[3] S. A. Teukolsky, Astrophys. J. 185, 635 (1973).

[4] S. Chandrasekhar and S. Detweiler, Proc. R. Soc. A 344, 441 (1975).

[5] F. Echeverria, Phys. Rev. D 40, 3194 (1989).

[6] O. Dreyer, B. J. Kelly, B. Krishnan, L. S. Finn, D. Garrison, and R. Lopez-Aleman, Classical Quantum Gravity 21, 787 (2004).

[7] E. Berti, V. Cardoso, and C. M. Will, Phys. Rev. D 73, 064030 (2006).

[8] S. Gossan, J. Veitch, and B. S. Sathyaprakash, Phys. Rev. D 85, 124056 (2012).

[9] J. Meidam, M. Agathos, C. Van Den Broeck, J. Veitch, and B. S. Sathyaprakash, Phys. Rev. D 90, 064009 (2014).

[10] E. Berti et al., Classical Quantum Gravity 32, 243001 (2015).

[11] E. Berti, A. Sesana, E. Barausse, V. Cardoso, and K. Belczynski, Phys. Rev. Lett. 117, 101102 (2016).

[12] V. Baibhav, E. Berti, V. Cardoso, and G. Khanna, Phys. Rev. D 97, 044048 (2018).

[13] V. Baibhav and E. Berti, Phys. Rev. D 99, 024005 (2019).

[14] J. Aasi et al., Classical Quantum Gravity 32, 115012 (2015).

[15] F. Acernese et al., Classical Quantum Gravity 32, 024001 (2015).

[16] B. P. Abbott et al. (LIGO Scientific Collaboration and Virgo Collaboration), Phys. Rev. Lett. 116, 061102 (2016). 
[17] B. P. Abbott et al. (LIGO Scientific Collaboration and Virgo Collaboration), Phys. Rev. Lett. 116, 241103 (2016).

[18] B. P. Abbott et al. (LIGO Scientific Collaboration and Virgo Collaboration), Phys. Rev. X 6, 041015 (2016).

[19] B. P. Abbott et al. (LIGO Scientific Collaboration and Virgo Collaboration), Phys. Rev. Lett. 118, 221101 (2017).

[20] B. P. Abbott et al. (LIGO Scientific Collaboration and Virgo Collaboration), Astrophys. J. 851, L35 (2017).

[21] B. P. Abbott et al. (LIGO Scientific Collaboration and Virgo Collaboration), Phys. Rev. Lett. 119, 141101 (2017).

[22] B. P. Abbott et al. (LIGO Scientifc Collaboration and Virgo Collaboration), arXiv:1811.12907.

[23] B. P. Abbott et al. (LIGO Scientific Collaboration and Virgo Collaboration), Phys. Rev. Lett. 116, 221101 (2016).

[24] W. Del Pozzo and A. Nagar, Phys. Rev. D 95, 124034 (2017).

[25] M. Cabero, C. D. Capano, O. Fischer-Birnholtz, B. Krishnan, A. B. Nielsen, A. H. Nitz, and C. M. Biwer, Phys. Rev. D 97, 124069 (2018).

[26] E. Thrane, P. D. Lasky, and Y. Levin, Phys. Rev. D 96, 102004 (2017).

[27] R. Brito, A. Buonanno, and V. Raymond, Phys. Rev. D 98, 084038 (2018).

[28] G. Carullo et al., Phys. Rev. D 98, 104020 (2018).

[29] G. Carullo, W. Del Pozzo, and J. Veitch, Phys. Rev. D 99, 123029 (2019).

[30] I. Kamaretsos, M. Hannam, S. Husa, and B. S. Sathyaprakash, Phys. Rev. D 85, 024018 (2012).

[31] L. London, D. Shoemaker, and J. Healy, Phys. Rev. D 90, 124032 (2014).

[32] M. Giesler, M. Isi, M. Scheel, and S. Teukolsky, arXiv: 1903.08284.

[33] A. Buonanno, G. B. Cook, and F. Pretorius, Phys. Rev. D 75, 124018 (2007).

[34] Y. Pan, A. Buonanno, A. Taracchini, L. E. Kidder, A. H. Mroué, H. P. Pfeiffer, M. A. Scheel, and B. Szilágyi, Phys. Rev. D 89, 084006 (2014).

[35] A. Taracchini, A. Buonanno, Y. Pan, T. Hinderer, M. Boyle, D. A. Hemberger, L. E. Kidder, G. Lovelace, A. H. Mroue, H. P. Pfeiffer, M. A. Scheel, B. Szilagyi, N. W. Taylor, and A. Zenginoglu, Phys. Rev. D 89, 061502(R) (2014).

[36] S. Babak, A. Taracchini, and A. Buonanno, Phys. Rev. D 95 , 024010 (2017)

[37] A. Bohe et al., Phys. Rev. D 95, 044028 (2017).

[38] S. Bhagwat, D. A. Brown, and S. W. Ballmer, Phys. Rev. D 94, 084024 (2016); 95, 069906(E) (2017).

[39] B. P. Abbott et al. (LIGO Scientific Collaboration and Virgo Collaboration), Phys. Rev. Lett. 116, 221101 (2016).

[40] The best-measured combination of mass and spin is defined as the linear combination of $M_{f}$ and $\chi_{f}$ corresponding to the principal components of the posterior distribution with the smallest associated eigenvalue.

[41] The spin-weighted spheroidal harmonics form the natural basis that arises in perturbation theory $[3,42,43]$. These functions are equivalent to the spin-weighted spherical harmonics in the limit of zero spin. For $\chi_{f}>0$, the spinweighted spheroidal harmonics can be written as superpositions of the spin-weighted spherical harmonics of the same $m$, but different $\ell[43,44]$. The effect of this mixing on the dominant $\ell=m=2$ spin-weighted spherical mode is negligible for a GW150914-like system [32].

[42] S. Teukolsky, Phys. Rev. Lett. 29, 1114 (1972).

[43] W. H. Press and S. A. Teukolsky, Astrophys. J. 185, 649 (1973).

[44] E. Berti and A. Klein, Phys. Rev. D 90, 064012 (2014).

[45] B. P. Abbott et al. (LIGO Scientific Collaboration and Virgo Collaboration), Phys. Rev. D 94, 064035 (2016).

[46] B. P. Abbott et al. (LIGO Scientific Collaboration and Virgo Collaboration), Classical Quantum Gravity 34, 104002 (2017).

[47] E. W. Leaver, Proc. R. Soc. A 402, 285 (1985).

[48] E. Berti, V. Cardoso, and A. O. Starinets, Classical Quantum Gravity 26, 163001 (2009).

[49] E. Berti, Ringdown, http://pages.jh.edu/ eberti2/ringdown/.

[50] LIGO Collaboration and Virgo Collaboration, https://www .gw-openscience.org.

[51] M. Vallisneri, J. Kanner, R. Williams, A. Weinstein, and B. Stephens, Proceedings, 10th International LISA Symposium: Gainesville, Florida, USA (2014) [arXiv:1410.4839]; J. Phys. Conf. Ser. 610, 012021 (2015).

[52] B. P. Abbott et al. (LIGO Scientific Collaboration and Virgo Collaboration), Phys. Rev. Lett. 116, 241102 (2016).

[53] See Supplemental Material at http://link.aps.org/ supplemental/10.1103/PhysRevLett.123.111102 for details about our inference implementation.

[54] V. Varma, D. Gerosa, L. C. Stein, F. Hebert, and H. Zhang, Phys. Rev. Lett. 122, 011101 (2019).

[55] J. Blackman, S. E. Field, M. A. Scheel, C. R. Galley, C. D. Ott, M. Boyle, L. E. Kidder, H. P. Pfeiffer, and B. Szilágyi, Phys. Rev. D 96, 024058 (2017).

[56] LIGO Scientific Collaboration and Virgo Collaboration, GWTC-1, https://doi.org/10.7935/82H3-HH23(2018).

[57] I. Verdinelli and L. Wasserman, J. Am. Stat. Assoc. 90, 614 (1995).

[58] A. Ghosh et al., Phys. Rev. D 94, 021101(R) (2016).

[59] A. Ghosh, N. K. Johnson-McDaniel, A. Ghosh, C. K. Mishra, P. Ajith, W. Del Pozzo, C. P. L. Berry, A. B. Nielsen, and L. London, Classical Quantum Gravity 35, 014002 (2018). 